\title{
Surface Corrosion Detection and Classification for Steel Alloy using Image Processing and Machine Learning \\ ${ }^{\text {"I }}$ Sanjay Kumar Ahuja, ${ }^{2}$ Manoj Kumar Shukla, ${ }^{3}$ Kiran Kumar Ravulakollu \\ ${ }^{1}$ AIIT, Amity University, Noida, India \\ ${ }^{2}$ ASET, Amity University, Noida, India \\ ${ }^{3}$ University of Petroleum \& Energy Studies (UPES), Dehradun, India \\ Email:SanjayAhuja@India.com, MKShukla@Amity.Edu,kiran007.r@gmail.com
}

Received: $09^{\text {th }}$ July 2018, Accepted: $14^{\text {th }}$ August 2018, Published: $31^{\text {st }}$ August 2018

\begin{abstract}
Usually Stainless steel is notable for corrosion resistance; however, corrosion can happen in the environment with low oxygen and high salinity environment. The visual approach for corrosion detection is completely dependent on the quality of images and specialist's knowledge and experience. Corrosion detection and analysis can be done either through chemical based approach or image processing based approach. Digital Image Processing is widely used non-destructive approach for detecting corrosion on the metal surface. It provides cost-effective, fast and reasonably accurate results provided appropriate algorithms have been used depending on the environmental conditions, corrosion type, lighting conditions etc. Several algorithms related to colour, texture, noise, clustering, segmentation, image enhancement, wavelet transformation etc. have been used in different combinations have been developed by different research groups for detecting corrosion using digital image processing techniques. This paper presents an adaptive self-learning approach for image processing based classification techniques for detecting different types of corrosion in steel. Corrosion has different colour and texture properties that can be analysed using image processing techniques. In the present paper, the research done in this field has been extensively reviewed to identify the challenges and the achievements in this area. For the present work, a combination of image segmentation approach along with machine learning approach has been used for classification of corrosion at the different concentration level of $\mathrm{HCl}$ and significantly reasonable results were obtained.
\end{abstract}

Keywords: Computer Vision, Image Processing, Corrosion, Segmentation, Wavelet, GLCM, SVM, Machine Learning

\section{Introduction}

Steel is an Iron alloy with other elements like Carbon, Chromium etc. in different percentage. The properties of steel e.g. hardness, ductility, and tensile strength varies with different percentage of element mass. It is widely used in construction and various other applications because of its high tensile strength, better corrosion resistance and low maintenance cost. Different types of corrosion are visually different and require different image processing algorithms. Below are the images of above mentioned corrosion types:

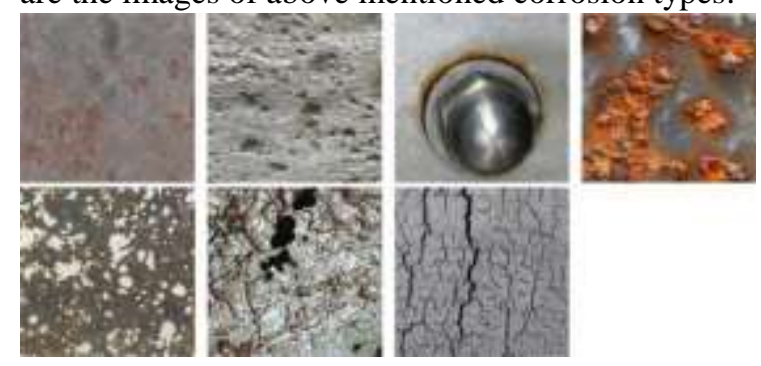

Fig. 1 Types of Corrosion: (a) General Corrosion; (b) Pitting Corrosion; (c) Crevice Corrosion; (d) Intergranular Corrosion; Galvanic Corrosion; (f) Erosion Corrosion and (g) Stress Corrosion.

The introduction of digital image processing opens expanded real life opportunities to sense in a variety of environments. While physical \& chemical tests are highly effective for corrosion detection, however are difficult to perform on large surface area. Digital Image processing based approaches are nondestructive and are economical to perform on large areas like ships, metal bridges, electric pole etc.

These provide fast, reasonably accurate and objective results. Various image processing techniques like image enhancement, noise removal, line detection, edge detection, registration, wavelet transformation, texture detection, morphological functions, colour analysis, segmentation, clustering and pattern recognition etc. are base functions that could be combined to detect corrosion on metal articles.

\section{Corrosion Detection Using Image Processing: Architecture}

This section provides the basic architecture for the corrosion detection using the image processing techniques. 


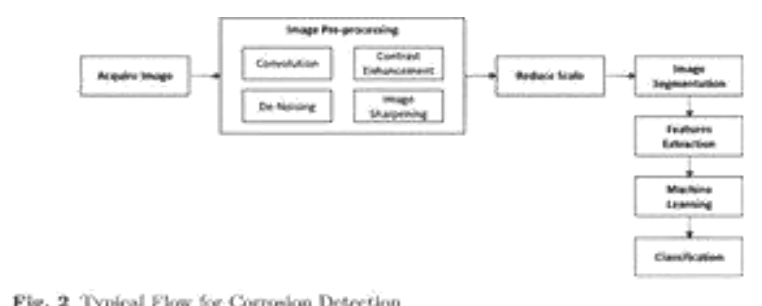

The typical steps involved in the process are:

1. Acquire Image: The very first step of image processing is essentially capturing of the images with the help of suitable camera with good resolution. The acquired images can be microscopic images or regular images from drone or CCTV.

2. Image Pre-Processing: The images acquired by microscope can be maintained to have better resolution and lightning conditions. However there may be images obtained through drone or CCTV in outdoor environment which may have high background noise and unwanted reflections. To use these images for computer vision, the noise need to be reduced using pre-processed by different filtering techniques. The various Image processing filters can be simple Median Filter OR complex filters like histogram equalization, Morphologic operations, Image stitching, Shadow removal etc.

3. Image Segmentation: Once the image is preprocessed, the next step is to identify the corrosion area in the image using Image Segmentation techniques such as Edge Detection, Region Growing, Thresholding and Clustering.

4. Feature Extraction: For image processing, the features need to be extracted from the image. The features may be related to colour, texture, shape etc. using Haar wavelets, Hough transform, Laplacian of Gaussian (LoG), Histogram of Oriented Gradients (HoG), Garbor filtering, Canny edge detection, Background subtraction and Wavelet transform etc. filters.

5. Machine Learning: Once the image attributes are collected, a suitable supervised machine learning algorithm can be implemented for classification and analysis. Alternatively, unsupervised machine learning or neural network based models. Both supervised and unsupervised approaches have their advantages and disadvantages. Depending on image dataset, a suitable approach can be used for creating a model that can be used for classification and analysis

6. Image Classification \& Analysis: In this step, classification is based on certain known features such as size, position, contour measurement, texture measurements etc. The image classifiers can be categorized based on Feature, Model and Pattern of the corroded area in the image.This step helps in detecting the corrosion in the images. Once corrosion is detected, the total corrosion area is calculated, to estimate whether the image is partially corroded or fully corroded to take appropriate action.

\section{Related Work}

Researchers have used different image processing techniques for the detection of the corrosion in the steel structures. The existing corrosion detection and analysis techniques can be categorized into different categories as:

\section{General Image Processing:}

Primarily the image processing techniques are based Filtering, Morphology and Edge based thresholding. Widely used techniques are:

- Colour based: Corroded images have high presence of red colour as compared to green and blue. Analysing the red colour in the image can be used for detecting the presence of corrosion in the images. As colour is dependent on lightning conditions, the other parameters e.g. Hue, Saturation and Value are widely used parameters for corrosion detection.

- Texture Analysis: The metal surface shows different roughness at different stages of corrosion growth. The texture or pattern of corroded area may be different at different region of images. The texture analysis can be used for segmentation of corroded and non-corroded parts.

- Wavelets: The Wavelet approach is based on Fourier Transform (FT) and overcome the shortcomings in FT. There are many different types of WTs, among those types Continuous Wavelet Transform (CWT) and Discrete Wavelet Transform (DWT) are widely used transforms.

2. Learning Based: Learning base approaches are based on supervised and unsupervised machine learning. Researchers have mainly used Support Vector Machine (SVM), AdaBoost, Artificial Neural Networks (ANN), Decision Tree and Deep Learning approaches.

- Classification using Support Vector Machine (SVM): In this technique, classification is based on the colour of the corroded area. The degree of the corrosion and the colour of corroded area enables to detect the corrosion area.

- Artificial Neural Network (ANN): ANN is identical to neurons in human brain. The neural network is formed by a set of neurons interconnected with each other through the synaptic weights. Knowledge is acquired by Neurons during the learning phase, which is utilized during the 
verification phase. The number of neurons and synaptic weights can be changed according to needs.

\section{Experiment}

Surface corrosion has been studied on Mild steel at different concentration of $\mathrm{HCl}$. The corrosion growth varies with the concentration of $\mathrm{HCl}$. A combination of different image segmentation algorithms has been used along with machine learning based approach for classification. The lab setup consists of Mild steel samples of $4 \mathrm{~cm} \times 4 \mathrm{~cm}$ placed under controlled environment for one month under different concentration of $\mathrm{HCl}$ as $1 \mathrm{MHCl}, 2 \mathrm{MHCl}, 200 \mathrm{ppm}$, $300 \mathrm{ppm}, 400 \mathrm{ppm}$ and $500 \mathrm{ppm}$.

\begin{tabular}{|l|c|l|l|}
\hline & \multicolumn{2}{|c|}{} & \multirow{2}{*}{ No. of Days } \\
\hline Concentration & Temperature & Humidity & No. \\
\hline $200 \mathrm{ppm}$ & $35^{0} c$ & 90 & 15 \\
\hline $300 \mathrm{ppm}$ & $35^{0} c$ & 90 & 15 \\
\hline $400 \mathrm{ppm}$ & $35^{0} c$ & 90 & 15 \\
\hline $500 \mathrm{ppm}$ & $35^{0} c$ & 90 & 15 \\
\hline $1 \mathrm{M}$ & $35^{0} c$ & 90 & 15 \\
\hline $2 \mathrm{M}$ & $35^{0} c$ & 90 & 15 \\
\hline
\end{tabular}

Table 1 Detection Method and References

It was observed that the corrosion growth was different at different concentration levels. Corrosion images had strong presence of red colour and texture was also different. Images were taken using electronic microscope and more than 1400 samples were prepared of the size of $256 \times 256$ pixels.

Below are the sample images at different concentration level:
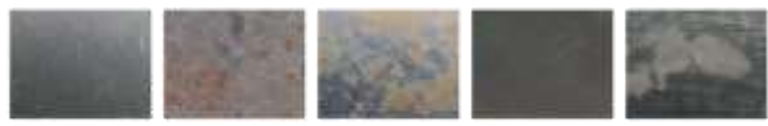

Fig. 3 Corrosion in Mild Steel: (a) Without Corrosion; (b) 100ppm HCl; (c) 200ppm HCl; (d) 300ppm HCl; (e) 400ppm HCl;

An Image processing and machine learning based approach has been proposed to classify the samples into aforesaid categories. The flow diagram for the proposed approach is as shown in Fig-4

The proposed work consists of a combination of colour and texture attributes as histogram and GLCM. The texture features are deployed using GLCM algorithm for 19 properties and 9 properties from histogram of Red, Green, Blue, Hue, Saturation and Value attributes.

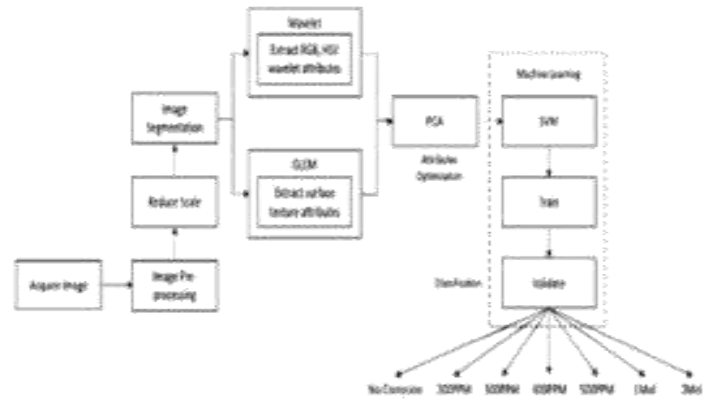

Fig. 4 Process Flow for Samples Classification Machine Learning

In this research, we proposed to use SVM for machine learning. SVM is a supervised machine learning based on statistical attributes of objects. SVM classifiers are used to classify pixels based on the texture feature vector assigned to each pixel. SVMs are generally two-class classifiers, however multiclass classification can also be achieved by oneversus-one and one-versus-all relationship. The most common technique is one -versus-rest classifiers, which has been used in this experiment to achieve classification among seven different categories.

These categories of images contain objects of a variety of texture that are arranged in horizontal and vertical directions. A set of 40 offsets that vary in distance have been taken to cover whole image. The plot for GLCM parameters with horizontal offset shows that there is correlation between GLCM parameters and pixel directions for different classes of images.

The GLCM parameters for different concentration of $\mathrm{HCl}$ is as shown in figure 5:
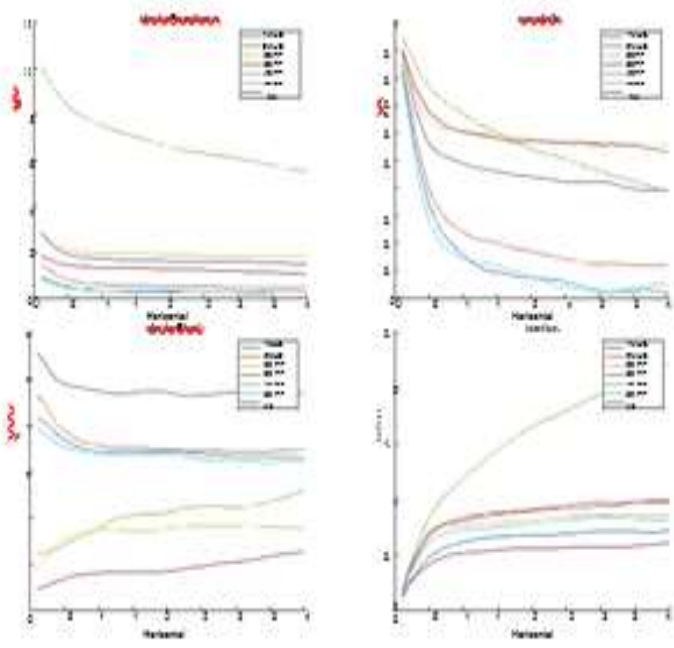

Fig. 5 (a)Cluster Prominence; (b) Correlation; (c) Cluster Shade; (d) Contrast 


\section{Results}

Overall 48 attributes per image were provided to machine learning algorithms for training and classification over a sample of more than 1400 images. The images were classified into No Corrosion, 200 ppm, 300 ppm, 400 ppm, 500 ppm, $1 \mathrm{M} \mathrm{HCl}, 2 \mathrm{M} \mathrm{HCl}$, based on the lab environment for different concentration of $\mathrm{HCl}$ solution for corrosion growth. The proposed approach has provided significantly acceptable results with $92.4 \%$ accuracy over classifying samples into these 7 classes.

\begin{tabular}{|l|l|l|l|l|}
\hline Type & Model & Accuracy & Prediction & Trainin \\
\hline & & & Speed & g Time \\
\hline & & & $($ obs/sec) & $($ sec $)$ \\
\hline Tree & Complex Tree & $77.20 \%$ & 6900 & 7.3351 \\
\hline Tree & Medium Tree & $69.60 \%$ & 15000 & 1.1009 \\
\hline Tree & Simple Tree & $58.10 \%$ & 14000 & 0.9514 \\
\hline SVM & Linear SVM & $86.60 \%$ & 3700 & 7.2516 \\
\hline SVM & Quadratic & $93.00 \%$ & 3400 & 5.6185 \\
\hline & SVM & & & \\
\hline SVM & Cubic SVM & $90.70 \%$ & 2900 & 5.7702 \\
\hline SVM & Fine Gaussian & $78.00 \%$ & 1900 & 8.4624 \\
\hline SVM & Medium & $89.10 \%$ & 2200 & 6.1713 \\
\hline & Gaussian & & & \\
\hline SVM & Coarse & $80.00 \%$ & 2500 & 5.202 \\
\hline & Gaussian & & & \\
\hline KNN & Fine KNN & $84.10 \%$ & 7000 & 1.3544 \\
\hline KNN & Medium Knn & $83.20 \%$ & 7700 & 0.83548 \\
\hline KNN & Coarse KNN & $66.40 \%$ & 7000 & 0.87792 \\
\hline KNN & Cosine KNN & $82.80 \%$ & 7700 & 0.92997 \\
\hline KNN & Cubic KNN & $83.00 \%$ & 780 & 6.2359 \\
\hline KNN & Weighted & $85.80 \%$ & 7300 & 0.8539 \\
\hline & KNN & & & \\
\hline KNN & Boosted KNN & $75.20 \%$ & 1600 & 21.29 \\
\hline Ensemble & Bagged Trees & $86.90 \%$ & 1600 & 10.062 \\
\hline Ensemble & Subspace & $83.90 \%$ & 1200 & 0.8233 \\
\hline & Discriminant & & & \\
\hline Ensemble & Subspace KNN & $67.50 \%$ & 570 & 10.122 \\
\hline Ensemble & RUSBoosted & $75.00 \%$ & 1700 & 14.667 \\
\hline & Trees & & & \\
\hline & & & \\
\hline Table 2 & Machine Lear & \\
\hline
\end{tabular}

Table 2 Machine Learning Performance Results

The proposed approach has provided significantly acceptable results with $92.4 \%$ accuracy over classifying samples into these 7 classes. The confusion matrix is as shown in figure 6

As the corrosion growth is not uniform across the samples, the experimental results indicated that $7.2 \%$ of the regions were wrongly classified with next class of the corrosion. The number of samples for $200 \mathrm{ppm}$, $300 \mathrm{ppm}$ and $400 \mathrm{ppm}$ were comparatively less, which resulted in false results, the machine learning algorithm could be better trained by providing more samples. The false results can be further optimized further by removing the outliers from the samples. The microscopic images were fine images with defined region of interest, which helped in achieving the model with high accuracy. Though SVM as supervised machine learning algorithm are known for less accuracy, however with the help of refined images and more than 40 attributes per image, it could classify the images with reasonably good results.

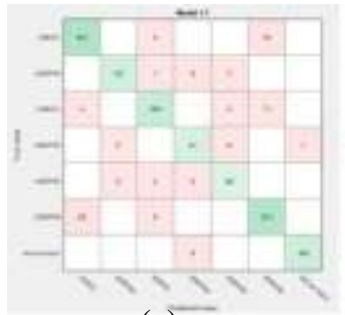

(a)

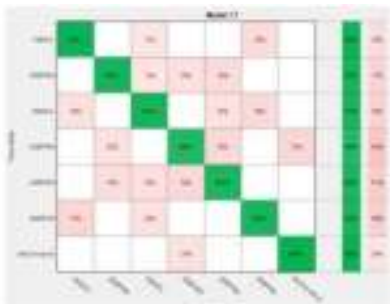

(b) (c)

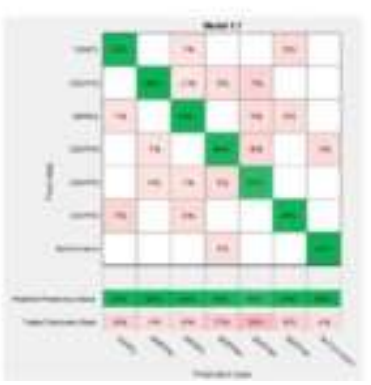

Fig. 6 (a) Confusion Matrix; (b) False Negative (c) True Positive

\section{Summary \& Conclusion}

The work has reported an average accuracy of $92.4 \%$ with field images of surface corrosion at 6 different level of $\mathrm{HCl}$ concentration. The texture features are deployed using GLCM algorithm for 19 properties. As an enhancement of the present work texture features were combined with other features like RGB colour and HSV properties of image to improve the accuracy.

\section{Future Work}

Currently the images taken for this research were fine images from microscope, however practically for outdoor scenario e.g. gas/oil pipelines it may not be feasible to have microscopic images. The outdoor images can be taken from drone or pig or even CCTV camera may be of low resolution. For future work, we suggest developing a representative data set for regular images instead of microscopic images, where image processing techniques can be applied for segmentation and clustering.

Also neural network based algorithms can be applied for automated solution for corrosion detection and analysis on metal articles. The metal articles can be small objects inside the museum or even large pipeline in the outdoor environment.

Also a mathematical model can be built for corrosion analysis at various stages and metal decay using 
supervised machine learning and analyse the validity and performance using time series analysis.

Computer vision based corrosion detection methods may also be used for the trend analysis and extrapolating the trends. This will help in taking timely preventive actions to stop the further deterioration of the metallic surfaces

\section{References}

1. Sundararaghavan V, Srivastava S. MicroFract: An image based code for micro structural crack path prediction. SoftwareX. pp. 94-97 (2017).

2. Ahuja, S.K. and Shukla, M.K. A Survey of Computer Vision Based Corrosion Detection Approaches. In International Conference on Information and Communication Technology for Intelligent Systems (pp. 55-63). (2017)

3. Aijazi, A.K., Malaterre, L., Tazir, M.L., Trassoudaine, L., Checchin, P.: Detecting And Analysing Corrosion Spots on the Hull of Large Marine Vessels Using Colored 3D Lidar Point Clouds. In: ISPRS Annals of Photogrammetry, Remote Sensing and Spatial Information Sciences pp. 153-160 (2016).

4. Petricca, L., Moss, T., Figueroa, G., Broen, S.: Corrosion Detection Using AI: A Comparison of Standard Computer Vision Techniques and Deep Learning Model. CCSEIT, AIAP, DMDB, MoWiN, CoSIT, CRIS, SIGL, ICBB, CNSA-2016 pp. 91-99, (2016).

5. Ortiz, A., Bonnin-Pascual, F., Garcia-Fidalgo, E.: Visual Inspection of Vessels by means of a Micro-Aerial Vehicle: an Artificial Neural Network Approach for Corrosion Detection. In: Robot 2015: Second Iberian Robotics Conference. Springer International Publishing, (2016).

6. Igoe, D., Parisi, A. V.: Characterization of the corrosion of iron using a smartphone camera. In: Instrumentation Science \& Technology $44.2 \mathrm{pp}$. 139-147 (2016).

7. Wang Y, Cheng G. Quantitative evaluation of pit sizes for high strength steel: Electrochemical noise, 3-D measurement, and image-recognition-based statistical analysis. Materials \& Design. pp. 176185 (2016).

8. Nascimento CC, Rodrigues SF, de Morais VM, Vilarinho LO. Methodology for corrosion evaluation in HAZ of $11 \%$-Cr ferritic stainless steel. Journal of Mechanical Science and Technology. pp.3805-3811 (2016).

9. Idris, S.A., Jafar, F.A., Jamaludin, Z., Blar, N.: Improvement of Corrosion Detection UsingVision System for Pipeline Inspection. In: Applied Mechanics \& Materials 761 (2015).

10. Son, H., Hwang, N., Kim, C., Kim, C.: Rapid and automated determination of rusted surface areas of a steel bridge for robotic maintenance systems. In: Automation in Construction $42 \mathrm{pp}$. 13-24 (2014).

11. Alkanhal, T.A.: Image Processing Techniques Applied for Pitting Corrosion Analysis. In: Entropy: International Journal of Research in Engineering and Technology, Volume: 03 Issue: 01 Jan (2014).

12. Idris, S.A., Jafar, F.A.: Image Enhancement Based on Software Filter Optimization for Corrosion Inspection. In: 2014 5th International Conference on Intelligent Systems, Modelling and Simulation. IEEE, (2014).

13. Bonnin-Pascual, F., Ortiz, A., Aliofkhazraei, D.M.: Corrosion Detection for Automated Visual Inspection. In: Developments in Corrosion Protection pp. 619-632 (2014).

14. Ranjan, R.K., Gulati, T.: Condition Assessment of Metallic Objects Using Edge Detection. In: International Journal of Advanced Research in Computer Science and Software Engineering Volume 4, Issue 5, May (2014)

15. Acosta, M.R.G., Daz, J.C.V., Castro, N.S.: An innovative image-processing model for rust detection using Perlin noise to simulate oxide textures. In: Corrosion Science 88 pp. 141- 151 (2014).

16. Margarita R. Gamarra Acosta, Juan C. Vlez Daz, An innovative image-processing model for rust detection using Perlin Noise to simulate oxide textures. Corrosion Science pp. 141- 151 (2014)

17. Fernndez-Isla, C., Navarro, P.J., Alcover, P.M.: Automated visual inspection of ship hull surfaces using the wavelet transform. In: Mathematical Problems in Engineering (2013).

18. Jahanshahi, M., Masri, S.: Effect of colour space, colour channels, and sub-image block size on the performance of wavelet-based texture analysis algorithms: An application to corrosion detection on steel structures. In: ASCE Intl. Workshop on Computing in Civil Eng. (2013)

19. Daira, R., Chalvedin, V., Boulhout, M.: Detection of Corrosion Processes in Metallic Samples of Copper by CND Control. In: Materials Sciences and Applications 4.04 pp. 238 (2013).

20. Sreeja, S. S, Jijina, K. P, Devi, J.: Corrosion Detection Using

Image Processing. In: International Research Journal of Computer Science Engineering and Applications Vol 2 Issue 4 April (2013)

21. Shen, H.K., Chen, P.H., Chang, L.M.: Automated steel bridge coating rust defect recognition method based on colour and texture feature. In: Automation in Construction $31 \mathrm{pp}$. 338- 356 (2013). 
Helix Vol. 8(5): 3822- 3827

22. Shen HK, Chen PH, Chang LM. Automated steel bridge coating rust defect recognition method based on colour and texture feature. Automation in Construction. pp. 338-356 (2013).

23. Chen, P.H., Shen, H.K., Lei, C.Y., Chang, L.M.: Support-vector-machine-based methodfor automated steel bridge rust assessment. In: Automation in Construction 23 pp. 9-19 (2012).

24. Liu, Z., Genest, M., Krys, D.: Processing thermography images for pitting corrosion quantification on small diameter ductile iron pipe. In: NDT \& E International 47 pp. 105-115 (2012).

25. Motamedi, M., Faramarzi, F., Duran, O.: New concept for corrosion inspection of urban pipeline networks by digital image processing. In: IECON 2012-38th Annual Conference on IEEE Industrial Electronics Society. IEEE, (2012).

26. Ji, G., Zhu, Y., Zhang, Y.: The corroded defect rating system of coating material basedon computer vision. In: Trans. Edutainment VIII, LNCS, vol. 7220, pp. 210-220. Springer (2012)

27. Nieves-Mendoza D, Gaona-Tiburcio C, Hervert Z, Mendez RC, Castro-Borges P, BorundaT, Zambrano Robledo P, AlmerayaCalderon F. Identifying Factors Influencing the Corrosion Rate of Steel Using Nonparametric Statistics. International Journal of Electrochemical Science. pp. 6343- 52 (2012).

28. Chen PH, Shen HK, Lei CY, Chang LM. Support-vector-machine-based method for automated steel bridge rust assessment. Automation in Construction. pp. 9-19 (2012).

29. Ghanta, S., Karp, T., Lee, S.: Wavelet domain detection of rust in steel bridge images."2011 IEEE International Conference on Acoustics, Speech and Signal Processing (ICASSP). IEEE, (2011).

30. Chen, P.H., Shen, H.K., Lei, C.Y., Chang, L.M.: Fourier-transform-based method for automated steel bridge coating defect recognition. In: Procedia Engineering 14 pp. 470-476 (2011).

31. Ghanta S, Karp T, Lee S. Wavelet domain detection of rust in steel bridge images. In Acoustics, Speech and Signal Processing (ICASSP), 2011 IEEE International Conference on pp. 1033-1036). (2011).

32. Zaidan, B.B., Zaidan, A.A., Alanazi, H.O., Alnaqeib, R.: Towards Corrosion DetectionSystem In: International Journal of Computer Science Issues, Vol. 7, Issue 3, No. 1, pp.33-36 May (2010) 33. Medeiros, F.N., Ramalho, G.L., Bento, M.P., Medeiros, L.C.: On the evaluation of texture and colour features for nondestructive corrosion detection. In: EURASIP Journal on Advances in Signal Processing 2010.1 pp.817473 (2010). 